\title{
Escala de Suporte Laboral: Correlação com suporte social, estresse e suporte organizacional
} Escala de Suporte Laboral: Correlation with social support, stress, and organizational support Escala de Suporte Laboral: Correlación con apoyo social, estrés y apoyo de la organización

\author{
Hugo Ferrari CARDOSO 1, a \\ Makilim Nunes BAPTISTA ${ }^{b}$ \\ Universidade Estadual Paulista “Júlio de Mesquita Filho”, São Paulo, SP, Brasila , Universidade São Francisco, \\ Itatiba, SP, Brasi ${ }^{b}$
}

ResumO Este estudo buscou evidenciar a validade com base na relação com variáveis externas para a Escala de Suporte Laboral (ESUL), bem como verificou as variáveis preditivas para o suporte organizacional. A amostra foi composta por 312 universitários que exerciam atividade profissional e responderam a quatro instrumentos (Escala de Suporte Laboral [ESUL], Questionário de Suporte Social [SSQ], Questionário de Percepção do Suporte Organizacional [QPSO] e Escala de Vulnerabilidade ao Estresse no Trabalho [EVENT]). Dos resultados, a ESUL se correlacionou positivamente com o SSQ e o QPSO e negativamente com a EVENT. Quanto à análise de regressão, as dimensões 1, 2 e 3 do QPSO formaram o modelo de maior explicação do suporte organizacional mensurado pela ESUL. Percebeu-se que a validade em questão pode ser evidenciada e novos estudos são sugeridos visando encontrar outros parâmetros psicométricos para a ESUL.

Palavras-chave:

Suporte organizacional; avaliação psicológica; evidência de validade.

\section{Abstract}

This study sought to demonstrate validity, based on the relationship with external variables, for the Escala de Suporte Laboral (ESUL), and identify the predictive variables for organizational support. The sample consisted of 312 college students with professional activities who responded to four instruments (ESUL, the Questionário de Suporte Social - SSQ, the Questionário de Percepsão de Suporte Organizacional - QPSO, and the Escala de Vulnerabilidade ao Estresse no Trabalho-EVENT). From the results, the ESUL was positively correlated with the $S S Q$ and the QPSO and negatively correlated with the EVENT. As for the regression analysis, the QPSO dimensions 1,2 , and 3 formed the model for further explanation of organizational support measured by the ESUL. The study demonstrated the validity of the scale in question and suggested further studies to search for other psychometric parameters for the ESUL.

Keywords:

Organizational support; psychological evaluation; evidence of validity.

Resumen

Este estudio buscó demostrar la validez basada en la relación con variables externas, de la Escala de Suporte Laboral (ESUL), y también encontró las variables predictivas para el apoyo organizacional. La muestra estuvo constituida por 312 estudiantes universitarios que ejercen la actividad profesional y respondieron a cuatro instrumentos (ESUL, el Questionário de Suporte Social - SSQ, el Questionário de Percepção do Suporte Organizacional - QPSO y la Escala de Vulnerabilidade ao Estresse no Trabalho - EVENT). Los resultados demostraron que el ESUL se correlacionó positivamente con el SSQ y el QPSO se correlacionó negativamente con el EVENT. En cuanto al análisis de regresión, las dimensiones 1, 2 y 3 de QPSO formaron el modelo para una explicación más detallada de apoyo organizacional medida por ESUL. Se observó, a partir del estudio, que la validez del instrumento en cuestión se puede demostrar y se sugieren más estudios en la búsqueda de otros

1 Endereço para correspondência: Av. Engenheiro Luiz Edmundo Carrijo Coube, № 14-01, Bairro: Vargem Limpa, Bauru, SP, Brasil 17033-360. Email: hfcardoso@fc.unesp.br 
parámetros psicométricos para ESUL.

Palabras-clave:

Apoyo de la organización; evaluación psicológica; pruebas de validez.

$\mathrm{O}$ suporte social é um construto amplo que engloba diversos contextos do relacionamento humano, destacando-se a família, o trabalho, as instituições de ensino, as instituições religiosas, entre outras. No entanto, há dificuldades em conceituá-lo e, consequentemente, avaliá-lo. De modo geral, há consenso entre os pesquisadores da área de que o suporte social é um construto multidimensional relacionado ao grau em que as necessidades sociais de uma pessoa são satisfeitas por meio de sua interação com os outros (Allemand, Schaffhuser, \& Martin, 2015; Cobb, 1976; Giurgescu et al., 2015; Guedea et al., 2006; Melrose, Brown, \& Wood, 2015; Rodriguez \& Cohen, 1998; Sarason, Levine, Basham, \& Sarason, 1983). Os mesmos autores ressaltam que a percepção do suporte social tem importante papel na redução do mal-estar, indicadores de depressão, estresse, inibição do desenvolvimento de doenças e, quando a pessoa está enferma, tende a desempenhar um papel positivo no processo de recuperação.

Com relação ao suporte organizacional, uma faceta do suporte social, de acordo com Siqueira e Gomide Jr. (2008), seu foco de estudo não se remete necessariamente a questões da saúde do trabalhador, mas sim, em uma primeira instância, à capacidade do suporte em explicar e predizer indicadores que interessam às organizações, como comprometimento organizacional, satisfação no trabalho, desempenho e produtividade. Nessa direção, o suporte organizacional está relacionado às percepções do trabalhador acerca da qualidade do tratamento que recebe da organização em retribuição ao esforço despendido no desenvolvimento de suas funções laborais (Eisenberger, Fasolo, \& Davis-LaMastro, 1990; Eisenberger, Huntington, Hutchison, \& Sowa, 1986; Hayton, Carnabuci, \& Esisenberger, 2012). Em outras palavras, a Percepção do Suporte Organizacional (PSO) está diretamente relacionada com as formas interacionais entre o trabalhador e a organização.

De acordo com Eisenberger et al. (1986), esse relacionamento, quando percebido de forma positiva pelos colaboradores, pode ser considerado, em grande parte, indicativo de um local agradável para se trabalhar, podendo desenvolver no empregado maior satisfação no desempenho de suas funções, bom humor, relacionamentos adequados com a equipe de trabalho, além do comprometimento no desempenho das funções laborais. Conforme complementam Kraimer, Seibert, Wayne, Liden e Bravo (2011), Tamayo e Tróccoli (2002) e Valcour, Ollier-Malaterre, Matz-Costa, Pitt-Catsouphes e Brown (2011), quando os colaboradores não percebem de forma positiva e leal o relacionamento com a organização, isso pode acarretar em um ambiente de trabalho menos comprometido e mais vulnerável ao estresse.

Por meio de uma revisão da literatura sobre a temática do suporte organizacional, Rhoades e Eisenberger (2002) enquadraram os resultados dos estudos analisados em categorias antecedentes e consequentes da PSO, sendo três consideradas antecedentes. A primeira refere-se à justiça de procedimentos, ou seja, formas equitativas de distribuição de recursos e de tratamento respeitoso aos empregados. A segunda refere-se ao suporte do suporte do supervisor, pois ele influencia, direciona e é responsável pelas condutas de seus subordinados no ambiente laboral. A terceira categoria, das recompensas e condições de trabalho, faz referência às práticas de recursos humanos, como pagamentos, promoções e desenvolvimento de autonomia no trabalho. Já as categorias consequentes da PSO estão relacionadas às reações e aos comportamentos dos empregados para com a organização. Nesse caso em específico, os autores destacam seis categorias decorrentes da percepção positiva de suporte: (a) comprometimento organizacional, (b) relacionamento afetivo no trabalho (p. ex., respeito, confiança), (c) envolvimento no trabalho (p. ex., lealdade), (d) desempenho, (e) diminuição de estresse e (f) desejo de permanência na instituição (Rhoades \& Eisenberger, 2002).

A respeito das interações entre colaboradores e organização, Seligman (2004) afirma que emoções positivas nesse contexto geralmente são percebidas como benéficas para ambas as partes. Caso contrário, emoções negativas evoluem para situações de jogos de poder em que há a percepção de que um lado deve ganhar e o outro perder. Nesse sentido, DeConinck (2010), Eisenberger, Stinglhamber, Vandenberghe, Sucharski e Rhoades (2002) e Estivalete e Andrade (2012) destacam que uma visão negativa por parte dos colaboradores pode acarretar em descontentamentos, erros, rotatividade e, futuramente, doenças, absenteísmo, principalmente decorrentes de situações estressoras no trabalho. 
Paschoal e Tamayo (2004) definem o estresse laboral como um processo em que as demandas de trabalho não estão em conformidade com as capacidades suportivas dos colaboradores. Esse construto pode ser observado por dois prismas, ou seja, visto sob a ótica dos colaboradores e sob a ótica da organização. De acordo com Genuíno, Gomes e Moraes (2009) e Gomes, Cruz e Cabanelas (2009), no primeiro caso, situações estressoras e descontentamentos no trabalho podem acarretar reações negativas e diminuição da qualidade de vida, além desses trabalhadores ficarem sujeitos de forma mais propensa a danos físicos e emocionais. $\mathrm{O}$ segundo prisma, na ótica da organização, de acordo com Calderero, Miasso e Corradi-Webster (2008), prevê que a presença de estresse nesse contexto pode acarretar menor produtividade, insatisfação dos colaboradores, dificuldades nos relacionamentos interpessoais e absenteísmo.

De acordo com os achados de alguns estudos, tais como os de Tamayo (2007), Coleta e Coleta (2008), Fernandes, Medeiros e Ribeiro (2008), Santos, Kitzberger, Morais, Lopes e Possamai (2008) e Suehiro, Santos, Hatamoto e Cardoso (2008), alguns fatores podem ser apontados como possíveis preditores de estresse laboral, entre eles falta de um ambiente de trabalho com tratamento digno, respeitoso e provedor de informações, pressão no trabalho, falta de autonomia e insegurança quanto à permanência no cargo. Os autores discutem o fato de algumas organizações estarem atentas a esses indícios, visando a manutenção de bem-estar aos funcionários, provavelmente preocupadas com sua produtividade e absenteísmo, entre outros fatores.

Para Baran, Shanock e Miller (2012) e Tamayo e Tróccoli (2002), situações estressoras e de esgotamento emocional no trabalho poderiam ser minimizadas por meio de melhorias no que diz respeito à qualidade do suporte organizacional. Partindo do princípio de que os suportes social e organizacional exercem papéis amortecedores em situações estressoras (Eisenberger et al., 1986; Gutierrez, Candela, \& Carver, 2012), e tendo como informação o fato de que no Brasil os estudos, e consequentemente a construção de instrumentos, sobre suporte organizacional não são robustos (Cardoso, 2010), um grupo de pesquisadores iniciou os estudos de construção e busca de parâmetros psicométricos para a Escala de Suporte Laboral - ESUL (Baptista, Cardoso, Santos, \& Noronha, 2013).

Nesse sentido, este estudo teve como objetivo principal verificar as correlações entre a ESUL e outros instrumentos que avaliam suporte social (Questionário de Suporte Social - SSQ), suporte organizacional (Questionário de Percepção de Suporte Organizacional - QPSO) e vulnerabilidade ao estresse laboral (Escala de Vulnerabilidade ao Estresse no Trabalho - EVENT), como forma de evidenciar a validade com base na relação com variáveis externas para a ESUL. Além disso, como objetivo específico, será testado um modelo com a análise de variáveis preditivas para o suporte organizacional mensurado por intermédio da ESUL.

\section{MÉTODO}

\section{Participantes}

A amostra foi composta por 312 trabalhadores que cursavam o ensino superior. Os estudantes eram provenientes de duas universidades privadas localizadas no interior do Estado de São Paulo. A idade dos participantes variou de 18 a 53 anos $(M=25,45 ; D P=7,45)$, com maior frequência do sexo feminino $(N=206$; $66 \%)$ e solteiros $(N=226 ; 72,4 \%)$. Com relação às organizações, classificadas de acordo com o Sebrae (2005), as maiores frequências de respostas foram na categoria pequenas empresas $(N=113 ; 36,2 \%)$ e prestadoras de serviços $(N=165 ; 52,8 \%)$. Já as ocupações mais destacadas foram aquelas enquadradas no grupo quatro $(N=$ 122; 39,1\%) da Categoria Brasileira de Ocupações (CBO, 2002), ou seja, relacionadas ao setor administrativo.

\section{Instrumentos}

\section{Escala de Suporte Laboral - ESUL}

A ESUL foi construída no Brasil por Baptista et al. (2013), com base na teoria de suporte organizacional de Eisenbereger et al. (1986), com ênfase na teoria de suporte social de Rodriguez e Cohen (1998). É composta por 24 itens, com pontuação variando de 0 a 72, na qual quanto maior a pontuação, maior o indício de percepção do suporte organizacional, sendo os itens distribuídos em quatro fatores: (a) Benefícios $(\alpha=0,84)$, (b) Interação colaborador e organização $(\alpha=0,78)$, (c) Interações entre colaboradores $(\alpha=0,73)$ e (d) Informação $(\alpha=0,81)$. 


\section{Questionário de Suporte Social - SSQ}

O SSQ é composto por 27 questões, e cada questão solicita duas respostas, número de fontes de suporte social percebido (SSQ-N) e satisfação com o suporte social recebido (SSQ-S), sendo que quanto maior a pontuação, em ambas as escalas, maior o indício de percepção do suporte social. O instrumento foi adaptado para o Brasil por Matsukura, Marturano e Oishi (2002), a partir do Social Support Questionnaire, construído por Sarason et al. (1983) e possui estudo de confiabilidade por intermédio de teste e reteste.

\section{Questionário de Percepção do Suporte Organizacional - QPSO}

O Questionário de Percepção de Suporte Organizacional foi desenvolvido e validado por Oliveira-Castro, Pilati e Borges-Andrade (1999). É composto por 46 itens, respondidos por meio de uma escala do tipo Likert de cinco pontos, com pontuação variando de 0 a 184. O instrumento possui estudos de evidências de validade de conteúdo e construto, assim como estudo de confiabilidade. Os 46 itens estão divididos em quatro fatores, com os respectivos índices de fidedignidade: (a) Gestão de desempenho $(\alpha=0,87)$; (b) Carga de trabalho ( $\alpha$ $=0,80$ ), cujos itens apresentam pontuações invertidas; (c) Suporte material $(\alpha=0,91)$; e (d) Valorização do trabalhador $(\alpha=0,83)$. Com relação à pontuação do questionário, nos fatores Gestão de desempenho, Suporte material e Valorização do trabalhador, quanto maior a pontuação, maior o indício de percepção do suporte organizacional. Já em relação ao fator Carga de trabalho, quanto maior a pontuação, menor o indício de percepção do suporte organizacional.

\section{Escala de Vulnerabilidade ao Estresse no Trabalho - EVENT}

A EVENT, construída por Sisto, Baptista, Noronha e Santos (2007), tem por objetivo avaliar a vulnerabilidade ao estresse do trabalhador quanto ao seu ambiente laboral. A escala é composta de 40 itens, respondidos por meio de uma escala tipo Likert de três pontos, com pontuação variando de 0 a 80 , indicando que quanto maior a pontuação, maior a vulnerabilidade ao estresse no ambiente laboral. A escala possui estudos de evidências de validade de conteúdo, construto, critério, estudos de confiabilidade e normatização. Os 40 itens estão divididos em três fatores: (a) Clima e funcionamento organizacional $(\alpha=0,88)$, (b) Pressão no trabalho $(\alpha=$ $0,85)$ e (c) Infraestrutura e Rotina $(\alpha=0,77)$.

\section{Procedimentos de coleta de dados e cuidados éticos}

Após a aprovação deste projeto pelo Comitê de Ética (Protocolo CAAE 0055.0.142.000-09), buscou-se a autorização dos professores para a aplicação dos instrumentos em salas de aula. Inicialmente, foram explicados os objetivos do projeto aos participantes, salientando que a participação não era obrigatória. Após, para aqueles que sinalizaram que trabalhavam, buscou-se o consentimento por meio do Termo de Consentimento Livre e Esclarecido (TCLE), impresso em duas vias, no qual constavam todas as informações pertinentes sobre a pesquisa.

A aplicação dos instrumentos ocorreu de forma coletiva, sendo que cada sala de aula teve em média 30 alunos (que trabalhavam e responderam aos instrumentos). Foi entregue um caderno contendo as duas vias do TCLE, ESUL, SSQ, QPSO e EVENT. A aplicação durou, em média, uma hora.

\section{Procedimentos de análise de dados}

Os dados foram analisados por meio da estatística inferencial utilizando-se correlação e regressão. Quanto à análise de correlação, foi utilizado o método de Pearson, enquanto a regressão foi linear e utilizou-se o método de análise por etapas.

\section{RESULTADOS}

Em relação às análises de correlação, informações que remetem ao objetivo de evidência de validade com base em variáveis externas, esperava-se que, entre ESUL e SSQ tais associações fossem positivas (uma vez que ambas se propõem a mensurar suporte), assim como as correlações entre ESUL e QPSO (ambas avaliam suporte organizacional). Em contrapartida, esperavam-se correlações negativas entre ESUL e EVENT (uma vez que, de acordo com informações já apresentadas na introdução deste estudo, quanto maior a percepção de suporte organizacional, menor tende a ser a percepção de estresse no trabalho). 
TABELA 1. Correlações entre ESUL e os demais instrumentos

\begin{tabular}{|c|c|c|c|c|c|c|}
\hline & & ESUL 1 & ESUL 2 & ESUL 3 & ESUL 4 & ESUL-T \\
\hline SSQ-N & $r$ & $-0,10$ & 0,01 & $0,15^{*}$ & 0,04 & 0,01 \\
\hline SSQ-S & $r$ & 0,03 & 0,10 & $0,15^{*}$ & 0,04 & 0,01 \\
\hline QPSO 1 & $r$ & 0,06 & $0,46^{\star *}$ & $0,35^{\star *}$ & $0,55^{\star *}$ & $0,50^{\star *}$ \\
\hline QPSO 2 & $r$ & $-0,08$ & $-0,36^{\star *}$ & $-0,17^{*}$ & $-0,36^{\star *}$ & $-0,39^{\star \star}$ \\
\hline QPSO 3 & $r$ & $0,17^{*}$ & $0,30^{\star *}$ & $0,29^{\star *}$ & $0,33^{\star *}$ & $0,35^{\star *}$ \\
\hline QPSO 4 & $r$ & 0,09 & $0,14^{*}$ & $0,17^{*}$ & $0,13^{*}$ & $0,17^{*}$ \\
\hline QPSO-T & $r$ & 0,10 & $0,18^{*}$ & $0,25^{\star *}$ & $0,27^{\star *}$ & $0,28^{\star *}$ \\
\hline EVENT 1 & $r$ & $-0,08$ & $-0,46^{\star *}$ & $-0,29^{* *}$ & $-0,51^{* *}$ & $-0,48^{\star *}$ \\
\hline EVENT 2 & $r$ & $-0,04$ & $-0,30^{\star *}$ & $-0,06$ & $-0,31^{\star *}$ & $-0,22^{\star \star}$ \\
\hline EVENT 3 & $r$ & 0,04 & $-0,24^{\star *}$ & $-0,31^{\star *}$ & $-0,23^{\star *}$ & $-0,22^{\star \star}$ \\
\hline EVENT-T & $r$ & $-0,04$ & $-0,41^{\star *}$ & $-0,22^{* *}$ & $-0,43^{\star *}$ & $-0,39 * *$ \\
\hline
\end{tabular}

Nota. * $p<0,005 ;$ ** $p<0,001$. SSQ-N - Número de pessoas concebidas como suportivas (SSQ); SSQ-S - Grau de satisfação com o suporte (SSQ); ESUL1 - Fator 1 - Benefícios; ESUL2 - Fator 2 - Interação colaborador e organização; ESUL3 - Fator 3 - Interação entre colaboradores; ESUL4 - Fator 4 Informação; ESULT - ESUL Total; QPSO1 - Fator 1 - Gestão de desempenho (QPSO); QPSO2 - Fator 2 - Carga de trabalho (QPSO); QPSO3 - Fator 3 - Suporte material (QPSO); QPSO4 - Fator 4 - Valorização do trabalhador (QPSO); QPSO-T - QPSO Total; EVENT1 - Fator 1 - Clima e funcionamento organizacional (EVENT1); EVENT2 - Fator 2 - Pressão no trabalho (EVENT); EVENT3 - Fator 3 - Infraestrutura e rotina (EVENT); EVENT-T - EVENT Total.

Como pode ser visualizado pela Tabela 1, conforme anteriormente salientado como hipótese, foram encontradas duas correlações de magnitude fraca, no sentido positivo, entre a ESUL e o SSQ. Com relação aos dados com significância estatística, a terceira dimensão da ESUL (Interação entre colaboradores) correlacionou-se de forma positiva com SSQ-N e SSQ-S.

Entre a ESUL e o QPSO também eram esperadas correlações positivas e com significância estatística, uma vez que ambos os instrumentos propõem-se a avaliar o suporte organizacional. Quanto às correlações com significância estatística, foram encontradas ao todo 17 correlações positivas e quatro negativas (cabe destacar que todas as negativas estão relacionadas ao segundo fator do QPSO - Carga de trabalho - que apresenta pontuação invertida), sendo 18 de magnitude fraca e três, moderada. Como era de se esperar, a ESUL, tanto no total como nas suas dimensões, apresentou correlação de sentido positivo com o QPSO. Em outras palavras, quanto maior a percepção de suporte organizacional em uma escala, maiores os dados referentes ao suporte organizacional na outra.

No que tange aos dados entre a ESUL e a EVENT, foram encontradas 15 correlações negativas e estatisticamente significativas, sendo 10 de magnitude fraca e cinco moderadas. Esse dado também era esperado, uma vez que a EVENT mensura os estressores laborais e, nesse sentido, de acordo com os dados obtidos, maior percepção de suporte organizacional na amostra esteve relacionada com menor percepção de estressores ocupacionais por parte dos trabalhadores. De certa forma, os dados obtidos no estudo corroboraram com a literatura, ou seja, em geral, suportes social e organizacional estão positivamente relacionados (Hayton et al., 2012; Siqueira \& Gomide Jr., 2008), bem como suporte organizacional pode ser considerado uma variável importante para que os trabalhadores percebam menos estressores no ambiente de trabalho (Baran et al., 2012; Eisenberger et al., 1986; Gutierrez et al., 2012; Tamayo \& Tróccoli, 2002). Pode-se dizer, nesses termos, que a ESUL foi capaz de evidenciar validade tendo como base algumas variáveis externas (instrumentos aplicados), como suporte social, suporte organizacional e estressores laborais.

Outro procedimento realizado foi análise de regressão linear, por meio do método por etapas, utilizando-se como variável dependente as pontuações da ESUL total. Já as variáveis independentes foram as pontuações do SSQ (número de pessoas percebidas como suportivas; grau de satisfação com o suporte), as dimensões do QPSO (1 - Gestão de desempenho; 2 - Carga de trabalho; 3 - Suporte material; 4 - Valorização no trabalho) e os fatores da EVENT (1 - Clima e funcionamento organizacional; 2 - Pressão no trabalho; 3 - Infraestrutura e rotina). Os resultados dessa análise podem ser visualizados na Tabela 2. 
TABELA 2. Regressão entre as variáveis

\begin{tabular}{|c|c|c|c|c|c|c|}
\hline \multirow[t]{2}{*}{ Modelo } & & \multirow{2}{*}{$\begin{array}{c}\begin{array}{c}\text { Coeficientes Não } \\
\text { padronizados }\end{array} \\
\text { B }\end{array}$} & & \multirow{2}{*}{$\begin{array}{c}\begin{array}{c}\text { Coeficiente } \\
\text { Padronizado }\end{array} \\
\beta\end{array}$} & \multirow[t]{2}{*}{$t$} & \multirow[t]{2}{*}{ Significância } \\
\hline & & & $S E$ & & & \\
\hline \multirow{4}{*}{3} & (Constante) & 27,363 & 3,706 & & 7,383 & 0,000 \\
\hline & QPSO1 & 0,272 & 0,084 & 0,295 & 3,220 & 0,001 \\
\hline & QPSO2 & $-0,304$ & 0,068 & $-0,314$ & $-4,472$ & 0,000 \\
\hline & QPSO3 & 0,228 & 0,091 & 0,203 & 2,495 & 0,013 \\
\hline
\end{tabular}

Nota. QPSO1 (Fator 1: Gestão de desempenho); QPSO2 (Fator 2: Carga de trabalho); QPSO3 (Fator 3: Suporte material).

Quanto aos resultados do modelo de regressão, maior percepção de gestão de desempenho foi a variável que mais pode predizer o alto suporte laboral por meio da ESUL. Em seguida, maior percepção de carga de trabalho foi preditivo para menor percepção de suporte e, logo após, o suporte material. Essas três variáveis formaram o modelo com maior poder preditivo $\left(R_{\text {ajustado }}^{2}=0,37\right)$ em relação ao suporte laboral avaliado pela ESUL. Vale ressaltar que o modelo com todas as variáveis (isto é, nove), a partir do método enter, é capaz de predizer 38\% da variável ESUL. Em outras palavras, o modelo final encontrado, com um terço das variáveis iniciais inseridas como preditoras, foi capaz de predizer praticamente a mesma porcentagem de variância do modelo com o total de variáveis preditoras. Em relação ao diagnóstico de colinearidade, o variance inflation fator (VIF) ficou entre 1,6 e 2,7, índice satisfatório, já que o mesmo não deve ser igual ou superior a 10. Já a tolerância (Tolerance) ficou entre 0,4 e 0,6, indicando que não há colinearidade entre as variáveis que traga prejuízo para a análise, já que valores aceitáveis devem ser iguais ou inferiores a 0,10 . Por último, em relação ao pressuposto de resíduos, a análise de Durbin-Watson demonstrou valor de 2,07, sugerindo baixa correlação entre os resíduos das variáveis no modelo, levando-se em consideração que valores próximos a 2 , em uma variação de 0 a 4, demonstram a inexistência de correlações indevidas positivas ou negativas.

\section{DISCUSSÃO}

A primeira discussão a ser feita neste artigo diz respeito ao seu objetivo principal, ou seja, a busca pela evidência de validade com base em variáveis externas para a ESUL. Tal procedimento, conforme visto anteriormente, ocorreu por meio da análise de correlação entre a ESUL e os demais instrumentos aplicados, quais sejam SSQ, QPSO e EVENT. Com base nas associações entre os instrumentos e em sua totalidade, os resultados corroboraram com os achados de outros autores, uma vez que a percepção de suporte organizacional, construto aferido pela ESUL, esteve relacionada de forma positiva ao suporte social, a outra escala de suporte organizacional (QPSO), e negativamente à vulnerabilidade de estresse laboral. Esses dados podem ser mais bem explicados pelas contribuições de Fredrickson (2001) e Passareli e Silva (2007), para os quais os ambientes sociais percebidos como benéficos tendem a ter como características o adequado relacionamento entre as pessoas, além do desenvolvimento de emoções positivas nesses contextos, o que diminuiria a probabilidade de percepção de um ambiente hostil e estressante.

Em outras palavras, tende a haver um consenso entre diversos autores, como, por exemplo, Airaksinen et al. (2015), Cobb (1976), Heintzelman e Bacon (2015), Miller, Esposito-Smythers e Leichtweis (2015), Rodriguez e Cohen (1998) e Sarason et al. (1983), de que o suporte social concebido como reforçador tende a estar associado à percepção de bem-estar, auxiliando, dessa forma, em situações estressoras específicas, como enfermidades e dificuldades no trabalho. Além disso, devido à maior quantidade de correlações significativas entre ESUL e SSQ-S, pode-se hipotetizar que, em se tratando de percepção de suporte, tanto laboral como social, a qualidade de apoio é vista como mais reforçadora do que meramente a percepção quantitativa de pessoas concebidas como suportivas.

No que concerne ao suporte laboral, as correlações foram positivas em relação ao suporte social, e negativa com vulnerabilidade ao estresse no trabalho. Em se tratando de um contexto específico (trabalho), alguns autores da área ressaltam que a percepção do suporte organizacional perpassa pela interação entre organização e colaborador, além da própria inter-relação entre os colaboradores da equipe de trabalho. Nesse sentido, quando essas interações são consideradas benéficas, o colaborador tende a perceber a organização como um local adequado para se trabalhar, uma vez que se sente bem, com humor positivo, valorizado, acolhido e recompensado, tanto financeira como emocionalmente (Eisenberger, Cummings, Armeli, \& Lynch, 1997; 
Neves \& Eisenberger, 2014; Yu \& Frenkel, 2013). Em outras palavras, e complementando os postulados de Haytoni et al. (2012), Hu, Wang, Yang e Wu (2014) e Tamayo e Tróccoli (2002), a percepção do suporte laboral tende a produzir no colaborador um senso de que ele é importante para a organização, resultando em um ambiente de trabalho mais agradável, com adequado relacionamento entre os funcionários, e podendo diminuir variáveis estressoras nesse contexto.

Por outro lado, na medida em que o colaborador não tem a percepção de suporte organizacional, de acordo com Eisenberger et al. (2002), Mitchell, Gagné, Beaudry e Dyer (2012), Rhoades e Eisenberger (2002) e Shoss, Eisenberger, Restubog e Zagenczyk (2013), ele pode se sentir mais estressado, não valorizado e descontente com o trabalho. De acordo com esses autores, o aumento de estresse no contexto organizacional pode ser atribuído, entre outros fatores, à precária percepção de suporte organizacional. Esse dado também corroborou com achados de outros autores, pois salientam que a presença intensa de estresse no trabalho pode influenciar negativamente tanto a produtividade como o relacionamento entre colaboradores e, em longo prazo, vir a acarretar o desenvolvimento de patologias, físicas e psicológicas, além do absenteísmo (Calderero et al., 2008; Genuíno et al., 2009; Gillet, Huart, Colombat, \& Fouquereau, 2013; Gomes et al., 2009; Scott, Zagenczyk, Schippers, Purvis, \& Cruz, 2014).

Por fim, a análise de regressão apresentou como variáveis preditoras de suporte laboral, utilizando como variável dependente a ESUL total, o primeiro e o terceiro fator do QPSO, que estiveram positivamente relacionados com a ESUL, e o segundo fator do QPSO, que esteve negativamente relacionado à escala. Para Rhoades e Eisenberger (2002), a exaustiva carga de trabalho, a pressão por produção e a insegurança quanto à permanência no emprego podem criar percepção negativa quanto ao suporte laboral, gerando negligência no trabalho, absenteísmo, entre outras consequências. Nessa direção, Eisenberger et al. (1997), Shen et al. (2014), Tuzun e Kalemci (2012) e Wang e Hsieh (2012) afirmam que a percepção do suporte organizacional perpassa por relacionamento pautado no bom senso entre organização e colaboradores, entretanto, se o funcionário se sente pressionado a produzir, ou seja, há sobrecarga de trabalho e baixa percepção de afetividade, de acordo com os autores, a tendência é que ele conceba o local de trabalho como sendo inadequado.

Apesar de não ser o objetivo deste artigo, é importante fazer referência à relação entre o QPSO e a ESUL, já que são escalas que parecem avaliar um construto similar, mas que possuem propriedades psicométricas, número de itens e descrição das dimensões singulares. É interessante notar que, das 25 correlações possíveis entre as dimensões e totais em ambos os instrumentos, 21 foram observadas entre as duas escalas com valores significativos, variando de fracos a moderados. Análises psicométricas posteriores, como, por exemplo, o emprego de técnicas bifatoriais, serão importantes para avaliar e comparar ambas as escalas do ponto de vista psicométrico, uma vez que elas podem explicar diferentes frações do construto. Independentemente de análises futuras, as escalas possuem algumas diferenças em termos de número de itens, variância explicada do construto, tipo de resposta, entre outras características, o que aumentaria a opção de uso para o profissional da área.

Nesse sentido, é importante que o psicólogo organizacional tenha opções de instrumentos diferentes, tanto do ponto de vista psicométrico como semântico, a fim de ampliar as possibilidades de avaliações nesse ambiente. Além disso, instrumentos com número diferente de itens e, consequentemente, com tempos de aplicação variáveis podem ser úteis em processos seletivos mais ou menos restritos nesse quesito. Independentemente da necessidade da comparação psicométrica das duas escalas em diversos quesitos (teoria clássica e moderna), e de não ter sido objetivo desta pesquisa, novos estudos psicométricos futuros se fazem necessários com ambas as escalas.

A pesquisa teve como propósito a busca de evidência de validade com base em variáveis externas (instrumentos que avaliam suporte social, organizacional e vulnerabilidade ao estresse no trabalho), bem como analisar as variáveis preditivas para o suporte organizacional (mensurado por meio da ESUL). Os dados obtidos foram coerentes aos objetivos propostos, no entanto, outros estudos de evidências de validade, com amostras probabilísticas e heterogêneas (p. ex., indústria, comércio, serviços) devem ser realizados para complementar os dados deste estudo.

Embora esta pesquisa tenha cumprido os objetivos propostos, algumas considerações devem ser feitas. Primeiro, no que tange a uma limitação do trabalho, amostras posteriores devem ser maiores e mais heterogêneas, além de utilizar somente estudantes universitários trabalhadores. Outra característica diz respeito à amostra de conveniência, em que os resultados devem ser tomados com cautela, limitando os achados. A amostra foi composta por universitários (que trabalhavam) e, nesse sentido, sugere-se que outros estudos sejam 
realizados utilizando a ESUL, com o intuito de aplicá-la também com outros instrumentos, tendo como propósito evidenciar outras formas de validade, e com amostras menos direcionadas, ou seja, não apenas com universitários, mas sim trabalhadores independentemente do nível de escolaridade de diversos segmentos organizacionais. Dessa forma, outras pesquisas com a escala devem ser realizadas para que evidências de validade diversas possam ser apresentadas.

\section{REFERÊNCIAS}

Airaksinen, J., Hakulinena, C., Elovainioa, M., Lehtimäkic, T., Raitakarie, O. T., Keltikangas-Järvinena, L., \& Jokela, M. (2015). Neighborhood effects in depressive symptoms, social support, and mistrust: Longitudinal analysis with repeated measurements. Social Science \& Medicine, 136, 10-16. doi:10.1016/2015.04.034

Allemand, M., Schaffhuser, K., \& Martin, M. (2015). Long-term correlated change between personality traits and perceived social support in middle adulthood. Personality and Social Psychology Bulletin, 41(3), 420-432. doi: 10.1177/0146167215569492

Baptista, M. N., Cardoso, H. F., Santos, A. A. A., \& Noronha, A. P. P. (2013). Escala de Suporte Laboral - ESUL (Manual técnico). São Paulo: Universidade São Francisco.

Baran, B. E., Shanock, L. R., \& Miller, L. R. (2012). Advancing organizational support theory into the twenty-first century world of work. Journal of Business and Psychology, 27(2), 123-125. doi: 10.1007/s10869-011-9236-3

Calderero, A. R. L., Miasso, A. I., \& Corradi-Webster, C. M. (2008). Estresse e estratégias de enfrentamento em uma equipe de enfermagem de pronto atendimento. Revista Eletrônica de Enfermagem, 10(1), 51-62.

Cardoso, H. F. (2010). Escala de Suporte Laboral (ESUL): Estudo das qualidades psicométricas (Dissertação de mestrado). Recuperado de http://livros01.livrosgratis.com.br/cp127817.pdf

Classificação Brasileira de Ocupações (2002). Classificação Brasileira de Ocupações. [Banco de dados]. Recuperado de http:// www.mtecbo.gov.br/cbosite/pages/saibaMais.jsf

Cobb, S. (1976). Social support as a moderator of life stress. Psychosomatic Medicine, 38, 300-314.

Coleta, A. S. M., \& Coleta, M. F. D. (2008). Fatores de estresse ocupacional e coping entre policiais civis. Psico-USF, 13(1), 5968.

Dancey, C., \& Reidy, J. (2006). Estatística sem matemática para psicologia: Usando SPSS para Windows. Porto Alegre: Artmed.

DeConinck, J. B. (2010). The effect of organizational justice, perceived organizational support, and perceived supervisor support on marketing employees' level of trust. Journal of Business Research, 63(12), 1349-1355. doi: 10.1016/j. jbusres.2010.01.003

Eisenberger, R., Cummings, J., Armeli, S., \& Lynch, P. (1997). Perceived organizational support, discretionary treatment, and job satisfaction. Journal of Applied Psychology, 82(5), 812-820. doi: 10.1037/0021-9010.82.5.812

Eisenberger, R., Fasolo, P., \& Davis-LaMastro, V. (1990). Perceived organizational support and employee diligence, commitment, and innovation. Journal of Applied Psychology, 75(1), 51-59. doi: 10.1037/0021-9010.75.1.51

Eisenberger, R., Huntington, R., Hutchison, S., \& Sowa, D. (1986). Perceived organizational support. Journal of Applied Psychology, 71(3), 500-507. doi: 10.1037/0021-9010.71.3.500

Eisenberger, R., Stinglhamber, F., Vandenberghe, C., Sucharski, I. L., \& Rhoades, L. (2002). Perceived supervisor support: Contributions to perceived organizational support and employee retention. Journal of Applied Psychology, 87(3), 565-573. doi: 10.1037/0021-9010.87.3.565

Estivalete, V. B., \& Andrade, T. F. (2012). A influência dos valores organizacionais na percepção de suporte organizacional com base na concepção dos colaboradores do setor bancário. Revista de Administração Mackenzie, 13(3), 214-244. doi: http:// dx.doi.org/10.1590/S1678-69712012000300010

Fernandes, S. M. B. A., Medeiros, S. M., \& Ribeiro, L. M. (2008). Estresse ocupacional e o mundo do trabalho atual: Repercussões na vida cotidiana das enfermeiras. Revista Eletrônica de Enfermagem, 10(2), 414-427.

Fredrickson, B. L. (2001). The role of positive emotions in positive psychology: The broaden-and-build theory of positive emotions. American Psychologist, 56(3), 218-226. doi: 10.1037/0003-066X.56.3.218

Genuíno, S. L. V. P., Gomes, M. S., \& Moraes, E. M. (2009). O estresse ocupacional e a síndrome de burnout no ambiente de trabalho: Suas influências no comportamento dos professores da rede privada do ensino médio de João Pessoa. Revista Anagrama, 3(2), 1-9.

Gillet, N., Huart, I., Colombat, P., \& Fouquereau, E. (2013). Perceived organizational support, motivation, and engagement among police officers. Professional Psychology: Research and Practice, 44(1), 46-55. doi: 10.1037/a0030066 
Giurgescu, C., Misra, D. P., Sealy-Jefferson, S., Caldwell, C. H., Templin, T., Slaughter-Acey, J. C., \& Osypuk, T. L. (2015). The impact of neighborhood quality, perceived stress, and social support on depressive symptoms during pregnancy in african american women. Social Science \& Medicine, 130, 172-180. doi: 10.1016/j.socscimed.2015.02.006

Gomes, A. R., Cruz, J. F., \& Cabanelas, S. (2009). Estresse ocupacional em profissionais de saúde: Um estudo com enfermeiros portugueses. Psicologia: Teoria e Pesquisa, 25(3), 307-318.

Guedea, M. T. D., Albuquerque, F. J. B., Tróccoli, B. T., Noriega, J. A.V., Seabra, M. A. B., Guedea, R. L. D. (2006). Relação do bem-estar subjetivo, estratégias de enfrentamento e apoio social em idosos. Psicologia: Reflexão e Crítica, 19(2), $301-308$. doi: http://dx.doi.org/10.1590/S0102-79722006000200017

Gutierrez, A. P., Candela, L. L., \& Carver, L. (2012). The structural relationships between organizational commitment, global job satisfaction, developmental experiences, work values, organizational support, and person-organization fit among nursing faculty. Journal of Advanced Nursing, 68(7), 601-614. doi: 10.1111/j.1365-2648.2012.05990.x

Hayton, J. C., Carnabuci, G., \& Eisenberger, R. (2012). With a little help from my colleagues: A social embeddedness approach to perceived organizational support. Journal of Organizational Behavior, 33, 235-249. doi: 10.1002/job.755

Heintzelman, S. J., \& Bacon, P. L. (2015). Relational self-construal moderates the effect of social support on life satisfaction. Personality and Individual Differences, 73, 72-77. doi: 10.1016/j.paid.2014.09.021

Hu, C., Wang, S., Yang, C., \& Wu, T. (2014). When mentors feel supported: Relationships with mentoring functions and protégés' perceived organizational support. Journal of Organizational Behavior, 35, 22-37. doi: 10.1002/job.1851

Kraimer, M. L., Seibert, S. E., Wayne, S. J., Liden, R. C., \& Bravo, J. (2011). Antecedents and outcomes of organizational support for development: The critical role of career opportunities. Journal of Applied Psychology, 96(3), 485-500. doi: 10.1037/ a0021452

Matsukura, T. S., Marturano, E. M., \& Oishi, J. (2002). O Questionário de Suporte Social (SSQ): Estudos da adaptação para o português. Revista Latino-americano de Enfermagem, 10(5), 675-681.

Melrose, K. L., Brown, G. D. A., \& Wood, A. M. (2015). When is received social support related to perceived support and wellbeing? When it is needed. Personality and Individual Differences, 77, 97-105. doi: 10.1016/j.paid.2014.12.047

Miller, A. S., Esposito-Smythers, C., \& Leichtweis, R. N. (2015). Role of social support in adolescent suicidal ideation and suicide attempts. Journal of Adolescent Health, 56, 286-292. doi: 10.1016/j.jadohealth.2014.10.265

Mitchell, J., Gagné, M., Beaudry, A., \& Dyer, L. (2012). The role of perceived organizational support, distributive justice and motivation in reactions to new information technology. Computers in Human Behavior, 28, 729-738. doi: 10.1016/j. chb.2011.11.021

Neves, P., \& Eisenberger, R. (2014). Perceived organizational support and risk taking. Journal of Managerial Psychology, 29(2), 187-205. doi: http://dx.doi.org/10.1108/JMP-07-2011-0021

Oliveira-Castro, G. A., Pilati, R., \& Borges-Andrade, J. E. (1999). Percepção de suporte organizacional: Desenvolvimento e validação de um questionário. Revista de Administração Contemporânea, 3(2), 29-51.

Paschoal, T., \& Tamayo, A. (2004). Validação da Escala de Estresse no Trabalho. Estudos de Psicologia, 9(1), 45-52.

Passareli, P. M., \& Silva, J. A. (2007). Psicologia positiva e o estudo do bem-estar subjetivo. Estudos de Psicologia Campinas, 24(4), 513-517.

Rhoades, L., \& Eisenberger, R. (2002). Perceived organizational support: A review of the literature. Journal of Applied Psychology, 87(4), 698-714. doi: 10.1037/0021-9010.87.4.698

Rodriguez, M. S., \& Cohen, S. (1998). Social support. In H. Friedman (Ed.), Encyclopedia of mental health (pp. 535-544). New York: Academic Press.

Santos, P. S. F., Kitzberger, J., Morais, A. C. F., Lopes, C. T., \& Possamai, D. J. (2008). Identificação de agentes estressores em trabalhadores de indústrias de Jaraguá do Sul. Anuário de Produção Acadêmica Docente, 12(2), 185-200.

Sarason, I. G., Levine, H. M., Basham, M. B., \& Sarason, B. R. (1983). Assessing social support: The Social Support Questionnaire. Journal of Personality and Social Psychology, 44(1), 127-139. doi: 10.1037/0022-3514.44.1.127

Scott, K. L., Zagenczyk, T. J., Schippers, M., Purvis, R. L., \& Cruz, K. S. (2014). Co-worker exclusion and employee outcomes: An investigation of the moderating roles of perceived organizational and social support. Journal of Management Studies, 51(8), 1235-1256. doi: 10.1111/joms.12099

Seligman, M. E. P. (2004). Felicidade autêntica: Usando a nova psicologia positiva para a realização permanente. Rio de Janeiro: Objetiva. 
Serviço Brasileiro de Apoio às Micro e Pequenas Empresas (2005). Boletim estatístico de micro e pequenas empresas. Brasília: SEBRAE. Recuperado de http://www.dce.sebrae.com.br/bte/bte.nsf/03DE0485DB219CDE0325701B004CBD01/\$File/ NT000A8E66.pdf

Shen, Y., Jackson, T., Ding, C., Yuan, D., Zhao, L., Dou, Y., \& Zhang, Q. (2014). Linking perceived organizational support with employee work outcomes in a chinese context: Organizational identification as a mediator. European Management Journal, 32, 406-412. doi:10.1016/j.emj.2013.08.004

Shoss, M. K., Eisenberg, R., Restubog, S. L. D., \& Zagenczyk, T. J. (2013). Blaming the organization for abusive supervision: The roles of perceived organizational support and supervisor's organizational embodiment. Journal of Applied Psychology, 98(1), 158-168. doi: 10.1037/a0030687

Siqueira, M. M. M., \& Gomide Jr., S. (2008). Suporte no trabalho. In M. M. M. Siqueira (Org.), Medidas do comportamento organizacional: Ferramentas de diagnóstico e de gestão (pp. 283-294). Porto Alegre: Artmed.

Sisto, F. F., Baptista, M. N., Noronha, A. P. P., \& Santos, A. A. A. (2007). Escala de Vulnerabilidade ao Estresse no Trabalho. São Paulo:Vetor Editora de Psicologia.

Suehiro, A. C. B., Santos, A. A. A., Hatamoto, C. T., \& Cardoso, M. M. (2008). Vulnerabilidade ao estresse e satisfação no trabalho em profissionais do programa de saúde da família. Boletim de Psicologia, 58(129), 205-18.

Tamayo, A. (2007). Impacto dos valores da organização sobre o estresse ocupacional. Revista de Administração Contemporânea - Eletrônica, 1(2), 20-33.

Tamayo, M. R., \& Tróccoli, B. T. (2002). Exaustão emocional: Relações com a percepção de suporte organizacional e com estratégias de coping no trabalho. Estudos de Psicologia, 7(1), 37-46.

Tuzun, I. K., \& Kalemci, R. A. (2012). Organizational and supervisory support in relation to employee turnover intentions. Journal of Managerial Psychology, 27(5), 518 - 534. doi: http://dx.doi.org/10.1108/02683941211235418

Valcour, M., Ollier-Malaterre, A., Matz-Costa, C., Pitt-Catsouphes, M., \& Brown, M. (2011). Influences on employee perceptions of organizational work-life support: Signals and resources. Journal of Vocational Behavior, 79(2), 588-595. doi: 10.1016/j. jvb.2011.02.002

Wang, Y., \& Hsieh, H. (2012). Organizational ethical climate, perceived organizational support, and employee silence: A crosslevel investigation. Human Relations, 66(6) 783-802. doi: 10.1177/0018726712460706

Yu, C., \& Frenkel, S. J. (2013). Explaining task performance and creativity from perceived organizational support theory: Which mechanisms are more important? Journal of Organizational Behavior, 34(8), 1165-1181. doi: 10.1002/job.1844 